\title{
əIs the Ocean Speeding Up? Ocean Surface Energy Trends
}

\author{
CARL WUNSCH ${ }^{\mathrm{a}, \mathrm{b}}$ \\ ${ }^{\mathrm{a}}$ Harvard University, Cambridge, Massachusetts; ${ }^{\mathrm{b}}$ Massachusetts Institute of Technology, Cambridge, Massachusetts
}

(Manuscript received 20 April 2020, in final form 28 August 2020)

\begin{abstract}
A recent paper by Hu et al. (https://doi.org/10.1126/sciadv.aax7727) has raised the interesting question of whether the ocean circulation has been "speeding up" in the last decades. Their result contrasts with some estimates of the lack of major trends in oceanic surface gravity waves and wind stress. In general, both the increased energy and implied power inputs of the calculated circulation correspond to a small fraction of the very noisy background values. An example is the implied power increase of about $3 \times 10^{8} \mathrm{~W}$, as compared to wind energy inputs of order $10^{12} \mathrm{~W}$. Here the problem is reexamined using a state estimate that has the virtue of being energy, mass, etc. conserving. Because it is an estimate over an entire recent 26 -yr interval, it is less sensitive to the strong changes in observational data density and distribution, and it does not rely upon nonconservative "reanalyses." The focus is on the energy lying in the surface layers of the ocean. A potential energy increase is found, but it is almost completely unavailable-arising from the increase in mean sea level. A weak increase in kinetic energy in the top layer $(10 \mathrm{~m})$ is confirmed, corresponding to an increase of order $1 \mathrm{~cm} \mathrm{~s}^{-1} \mathrm{yr}^{-1}$ over 26 years. An estimate of kinetic energy in the full water column shows no monotonic trend, but the changes in the corresponding available potential energy are not calculated here.
\end{abstract}

KEYWORDS: Ocean circulation; Climate variability; Decadal variability; Oceanic variability; Trends

\section{Introduction}

Detection of what can be called "trends" in observed oceanic elements of the climate system is greatly complicated by a number of nearly intractable problems, many related to the multidecadal nature of the phenomena (e.g., Wunsch 2018). These problems include 1) the very thin database in early years, 2) the changing technologies over the decades, and 3) shifts in the geography of coverage in a very heterogeneous fluid. Other problems cannot be neglected and include calibration accuracy changes, differing assumptions about the statistics in interpolation/extrapolation schemes, and poor knowledge of the low-frequency natural variability. When models are used in the calculations, system drift owing to lack of model equilibrium and both systematic and random-walk errors also intrude.

A recent paper by $\mathrm{Hu}$ et al. (2020, hereafter $\mathrm{H} 20)$ raises the interesting question of whether in recent decades the largescale ocean circulation has become more intense (faster). ${ }^{1}$ The question is sufficiently intriguing and important enough to warrant further examination of some of the underlying elements that go, generically, into such estimates. Prior experience suggests caution when interpreting their conclusions:

\footnotetext{
${ }^{1}$ Interpreting the result as part of global warming, and with speculations as to the implications of a faster ocean circulation, this paper attracted some wider media attention (e.g., Washington Post, 5 Feb 2020, https://www.washingtonpost.com/climate-environment/ 2020/02/05/worlds-oceans-are-speeding-up-another-mega-scaleconsequence-climate-change/).
}

¿ Denotes content that is immediately available upon publication as open access.

Corresponding author: Carl Wunsch, cwunsch@fas.harvard.edu; carl.wunsch@gmail.com
1) When trends appear associated with a major change in technology, or associated with a change in numbers or coverage, the result is always suspect, although coincidence is also always possible. So, for example, H20 showed an apparent increase in sea surface wind speeds about 1979 just as satellite coverage of the global atmosphere became available. The major change in slope of most of their estimates of monthly ocean kinetic energy begins near 1992 when the first high-accuracy altimetric satellite dataset became available (their Fig. 1).

2) H20 relied heavily for their estimates on what are known as "reanalyses." Characteristically, these products are generated by sequentially forcing the climate system (or its subsystems of atmosphere, ocean, ice) toward observations at "analysis times" typically separated by $6 \mathrm{~h}$ in weather forecasting (Kalnay 2003, chapter 5). A major consequence of this predictor-corrector procedure is the failure of conservation of physical properties such as energy, mass, total water, salt, etc. Furthermore, if the density of a particular measurement type, such as the Argo profiles, increases by a factor of 4 (e.g., Li et al. 2017), it is easy to understand how extra energy may be injected into the system over time (Dee et al. 2011, their Fig. 20) for any model-based procedure tending to underestimate variability (e.g., Laepple and Huybers 2014). Measured kinetic energy in a geostrophically balanced field can be a strong function of distance involved, particularly in power-law spectrum fluids. Bengtsson et al. (2004) published a paper with the provocative title "Can climate trends be calculated from reanalysis data?" and their answer was "no.",

\footnotetext{
${ }^{2}$ It is worth quoting a part of their abstract: "Total kinetic energy shows an increasing global trend. Results from data assimilation experiments strongly suggest that this trend is also incorrect and mainly caused by the huge changes in the global observing system in 1979; no significant change in global kinetic energy can be found."
} 
TABLE 1. Approximate values of oceanic energy reservoirs. Values are totals unless stated otherwise. Note that $10^{18} \mathrm{~J}$ is 1 exajoule (EJ). "Reference" value is to provide a rough order of magnitude of the kinetic energy change if the whole water column changed by $1 \mathrm{~cm} \mathrm{~s}$. Values here are expected accurate to within an order of magnitude or better and various estimates do exist in the wider literature. Lowest value of the de Verdière et al. (2018) estimates is used here. A few other reference values are provided for context. Values omitted entirely include the internal and chemical energies, that of floating ice, and that of the entire oceanic biomass-see Dewar et al. (2006) and Katija (2012).

\begin{tabular}{lcl}
\hline \hline \multicolumn{1}{c}{ Energy reservoir } & Joules & \multicolumn{1}{c}{ Reference } \\
\hline PE general circulation & $2 \times 10^{25}$ & Oort et al. (1994) \\
Available PE general circulation & $(200-700) \times 10^{18}$ & de Verdière et al. (2018) and Huang (2005) \\
Geostrophic variability & $10 \times 10^{18}$ & FW \\
Surface gravity waves & $1.5 \times 10^{18}$ & Rascle et al. (2008) \\
Internal waves & $1.4 \times 10^{18}$ & Munk (1981) \\
Equilibrium tide & $0.05 \times 10^{18}$ & Munk and MacDonald (1960) \\
Barotropic tide & $0.36 \times 10^{18}$ & Ray and Egbert (2018) \\
Reference KE, $1 \mathrm{~cm} \mathrm{~s}^{-1}$, barotropic & $0.7 \times 10^{18}$ & Analytic order of magnitude \\
Inferred change in general circulation & $0.2 \times 10^{18}$ & H20 \\
\hline
\end{tabular}

3) $\mathrm{H} 20$ found an increase in water column total kinetic energy of order $2 \times 10^{17} \mathrm{~J}$ over 20 years (the time interval in the various products does vary). This change is a minute fraction of the energy thought to exist in the quasi-statistically steady oceanic flow. The corresponding required extra input of power is $10^{16} \mathrm{~J} \mathrm{yr}^{-1}$ or $\approx 3.2 \times$ $10^{8} \mathrm{~J} \mathrm{~s}^{-1}$, which in turn is a very small fraction of the $\approx 1 \times$ $10^{12} \mathrm{~W}$ or larger, thought required to sustain the oceanic steady state. Table 1 is a compilation of some of what is known about resident energy reservoirs, and Table 2 lists estimates of the power required to sustain oceanic motions [see Ferrari and Wunsch (2009) and Ferrari and Wunsch (2010, hereafter FW) for a more general account]. Values in the tables are displayed in graphical form in Fig. 1, including the H20 numbers. Even if the estimates shown are too large by an order of magnitude, the H20 inferred change in both resident energy and power input remain relatively very small.

Background eddy energy in the ocean is thought to be about $10^{19} \mathrm{~J}$ (10 exajoules, EJ; see, e.g., Ferrari and Wunsch $2009 ; \mathrm{FW})$. A $1 \%$ change in that value would roughly equal the H20 estimate, but a claim that eddy or any other component of energy is today known to that level of accuracy would be surprising. One measure of how well the ocean is understood today can be derived from the accuracies of estimates of its major energy reservoirs and supporting sources and sinks.

Apart from the specific $\mathrm{H} 20$ calculations, questions about changing oceanic energies, causes and effects, are both interesting and important and become a vehicle for discussion of a wide variety of problems. Several approaches exist to determining, understanding and attributing the origins of total energy changes. They include changes in

1) forcing rates (transferred power),

2) dissipation rates,

3) transfers from unresolved reservoirs, and

4) direct calculation of a change in the system energy.

$\mathrm{H} 20$ rely primarily on item 4 with some discussion of item 1 . Below, another estimate from item 4 will be made from an energy conserving state estimate [Estimating the Circulation and Climate of the Ocean (ECCO); Fukumori et al. 2018, 2019].

\section{Context}

Some zero-order comments about the various approaches may be helpful in interpreting what follows (see FW; Olbers et al. 2012, p. $353+$ ).

\section{a. Changing forcing}

The surface boundary layer, part of the air-sea interface, is a complicated region. A qualitative difficulty in interpreting the energy input from winds arises immediately in Table 2: the apparent power input from the wind to the ocean is dominated by the energy input into surface waves, with the part available to the large-scale general circulation almost indistinguishable from zero [see also Wang et al. (2006) and the discussion by von Storch et al. (2007) of boundary layer leakage into the interior]. Other studies have attempted to evaluate the buoyancy exchanges-made difficult by their dependence upon the

TABLE 2. Power sources for oceanic motions including some provided only for reference purposes. Omitted is the buoyancy work energies. Note $10^{12} \mathrm{~W}$ is 1 terawatt (TW). Again, values are believed reliable to better than an order of magnitude.

\begin{tabular}{|c|c|c|}
\hline Power source rates & Watts & Reference \\
\hline Wind total & $70 \times 10^{12}$ & FW \\
\hline $\begin{array}{l}\text { Wind to surface } \\
\text { gravity waves }\end{array}$ & $68 \times 10^{12}$ & Rascle et al. (2008) \\
\hline $\begin{array}{l}\text { Wind to general } \\
\text { circulation }\end{array}$ & $1-3 \times 10^{12}$ & Rimac et al. (2016) \\
\hline Wind to internal waves & $0.2 \times 10^{12}$ & Thorpe (2005) \\
\hline $\begin{array}{l}\text { Tides (barotropic and } \\
\text { baroclinic) }\end{array}$ & $3.5 \times 10^{12}$ & Munk and Wunsch (1998) \\
\hline Geothermal & $0.1 \times 10^{12}$ & Davies and Davies (2010) \\
\hline Net warming & $0.5 \times 10^{12}$ & Meyssignac et al. (2019) \\
\hline $\begin{array}{l}\text { Biological } \\
\text { productivity (solar) }\end{array}$ & $63 \times 10^{12}$ & Dewar et al. (2006) \\
\hline Inferred power increase & $3.2 \times 10^{8}$ & $\mathrm{H} 20$ \\
\hline
\end{tabular}



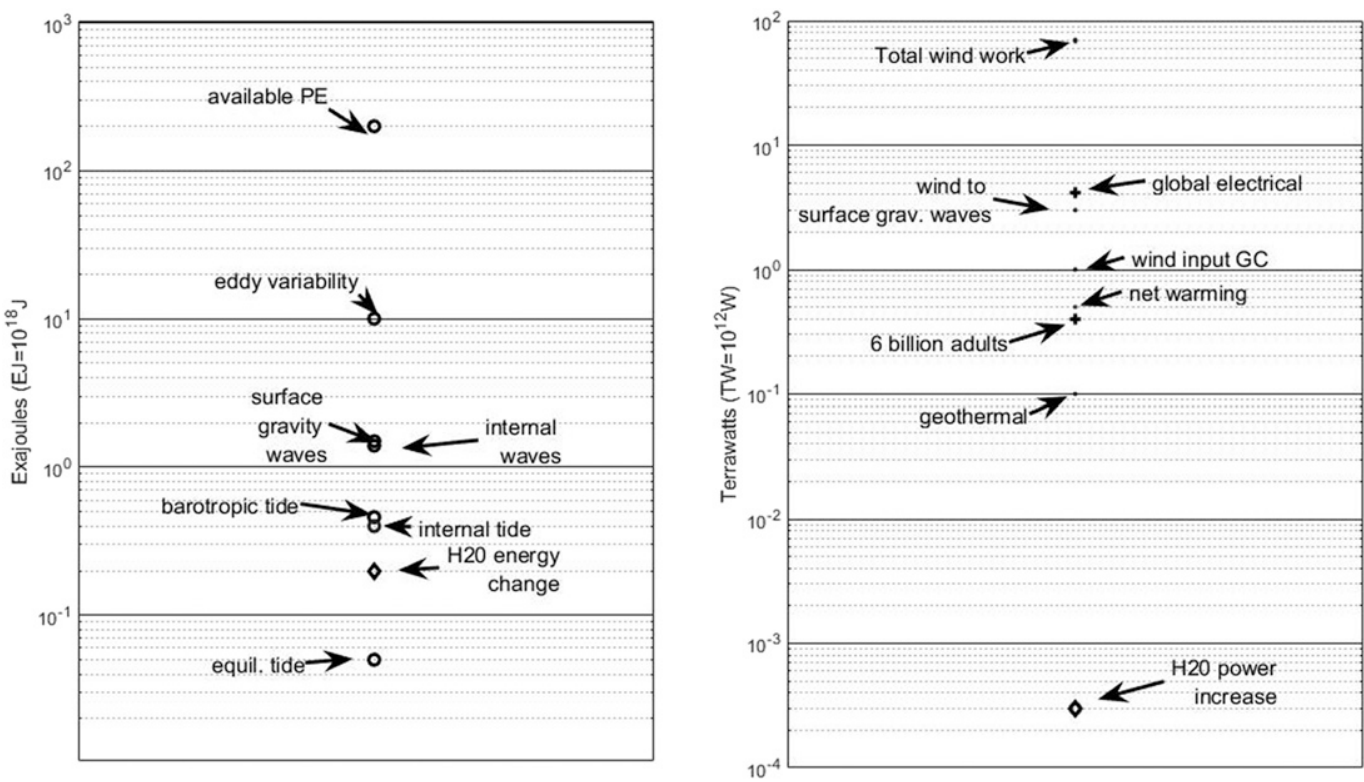

FIG. 1. (left) Estimated energy reservoirs in exajoules (EJ) and (right) power inputs in terawatts (TW) as listed in Tables 1 and 2. Note the logarithmic scales.

simultaneously occurring mechanical wind working [see references to controversy in Tailleux (2013) and Roquet (2013)]. Tides, whose long-term forcing amplitude changes are uniquely well understood, are thought to primarily affect abyssal and boundary mixing rates.

An inference that the wind field is placing increasing energy into the oceanic general circulation thus immediately suggests the question of whether the oceanic gravity wave field has not also amplified? Wave physics are sufficiently complicated by themselves that a wind-field increase does not necessarily imply a wave energy increase, but if one is observed, it would be useful supporting evidence. A recent study of winds and waves, Young and Ribal (2019), relied upon the comparatively homogeneous and quasi-global satellite datasets 1985-2018. They found little or no trend-like features in significant wave height and a small, marginal, increase (only at estimated $90 \%$ confidence) in wind speeds. (They do note that the increasing numbers of satellites with time can produce a positive bias in their results by better sampling of storms.) Issues do arise (Rascle and Ardhuin 2013; F. Ardhuin 2020, personal communication) from the technical details of each satellite system. Whatever has happened to the energy dominant wave field over the last $30+$ years appears to be subtle. Dissenters exist, e.g., Reguero et al. (2019), but see the caveats in Abdalla and Janssen (2018). Rascle et al. (2008) estimated the resident gravity wave energy as $1.5 \times 10^{18} \mathrm{~J}(1.5 \mathrm{EJ})$, with an energy input of about $70 \times 10^{12} \mathrm{~W}(70 \mathrm{TW})$, both useful reference values.

\section{b. Changing dissipation}

Dissipation rates of kinetic and potential energy in the ocean are primarily known in global value through the inference that the ocean is in a near-steady state, with dissipation necessarily then being equal to energy input. Spatial-scale transfers are the subject of a large and complicated literature, and the details of dissipation at topographic boundaries on all scales are the subject of a rapidly growing number of studies. A useful summary of time scales can be derived by dividing the reservoir magnitudes by the power inputs, where relevant, as depicted in Fig. 1 and Tables 1 and 2. Thus, all of the energy in the surface wave field is apparently dissipated in about $6 \mathrm{~h}$ (N. Rascle 2020, personal communication). Where that energy goes, in the presence of the complexities of wave breaking, is unclear. Similarly, the available potential energy (PE) of the general circulation in the de Verdière et al. (2018) computation, using their lower bound, if divided by an input of $1 \mathrm{TW}$, produces a time scale of $2 \times 10^{20} \mathrm{~J} / 10^{12} \mathrm{~J} \mathrm{~s}^{-1} \approx 6$ years as a minimum. Locating that dissipation globally is another serious challenge.

\section{c. Unresolved reservoirs}

Interpretation of the physics of "open" systems, ones where important sources or sinks of energy of a system are unknown, is almost impossible. Thus measurements e.g., of the energetics of the upper layers of the ocean, even if complete, are "open" to the extent that energy exchange can occur at unknown rates with the deeper ocean. Even with near-perfect understanding of exchanges with the atmosphere, negligible exchanges with depths have to be demonstrated or assumed over any finite interval being discussed.

Most of the kinetic energy in the ocean lies in the geostrophically balanced eddy field (FW). It has been known for many years that it varies spatially by at least two orders of magnitude. Indications are (e.g., Stammer et al. 2006, and others) that it also varies at least seasonally and probably interannually as well. Coupling between the eddy field and the larger-spatial-scale circulation is a long story involving numerous instabilities and scale interactions, and it seems fair to conclude that little or nothing is known about the interdecadal 
variability of these exchanges on a global basis (some regional studies exist, e.g., Qiu et al. 2017). Resolving the balanced eddy field, plus such features as the submesoscale and internal waves, has lain beyond the numerical capacity of global models run to quasi steady state.

\section{d. Direct estimates}

\section{REANALYSES}

Regional measurements of oceanic velocity and stratification variations lead directly to estimates of kinetic and potential energies. Piecing those together to produce global interdecadal changes has thus far been very difficult, largely because of the paucity of early data. Precision altimetry, dating to 1992, has permitted some discussion of parts of the energetics on a global basis (e.g., Wunsch 1997; Wang et al. 2013), but the direct measurements are restricted to surface height variations. Beginning in the early 2000s, the Argo profiles (e.g., Roemmich et al. 2009) have permitted estimates of global upper-ocean stratification and kinetic energy changes, albeit with the changing numbers and deployment-region pattern problems already alluded to.

A more direct attack on the problem of global changes can be made through the use of reanalyses - the central methodology of H20. Here the available observations are combined with the dynamics of a general circulation model (GCM), with the GCM acting as a dynamical interpolator in space and time. To understand the oceanic issues in particular, and recalling the Bengtsson et al. (2004) reference alluded to above, consider the elements of any oceanic "state vector" $\mathbf{x}(t)$. The state vector (a terminology originating in control theory) constitutes here the vector of properties including three components of velocity, temperature, salinity, and pressure, required by a model to take one discrete time step into the future time $t+\Delta t$ and usually written with an operator $\mathcal{L}($.$) in the "prediction" form,$

$$
\mathbf{x}(t+\Delta t,-)=\mathcal{L}[\mathbf{x}(t,-), \boldsymbol{\Gamma}(t) \mathbf{q}(t)],
$$

where $\boldsymbol{\Gamma}(t) \mathbf{q}(t)$, a matrix and vector respectively, represents all of the external elements required to run the model (initial conditions, boundary conditions, forcing, and model parameters) one time step. The minus sign is introduced here in the argument $\mathbf{x}(t)$ as a conventional label implying that direct measurements of any elements, or any combination of elements of $\mathbf{x}(t)$, have not been used in the predicted value since $t=0$. Such a model, if used for climate time scales, is required to satisfy basic conservation principles (energy, mass, salt, etc.) in the general sense that global changes are balanced by known and specified external forcing fields and internal sources and sinks.

Consider for example, the mechanical energy in the state $[\mathrm{PE}$ and kinetic energy (KE)] at any location $\mathbf{r}$ at time $t$,

$$
\begin{aligned}
\mathcal{E}\left(\mathbf{r}_{i}, t,-\right) & =\operatorname{PE}[\mathbf{x}(t,-), \mathbf{r}, t]+\operatorname{KE}[\mathbf{x}(t,-), \mathbf{r}, t], \\
\mathcal{E}_{\text {global }}(t,-) & =\left\langle\left\langle\mathcal{E}\left(\mathbf{r}_{i}, t-\right)\right\rangle\right\rangle,
\end{aligned}
$$

where the elements of the potential and kinetic energies are subelements of $\mathbf{x}(t)$ (typically vertical displacements and velocity components). A double angle bracket is used to indicate a global volume integral.
Most models will satisfy a conservation equation such as

$$
\begin{aligned}
\frac{\partial \mathcal{E}_{\text {global }}(t,-)}{\partial t} & \approx \frac{\mathcal{E}_{\text {global }}(t,-)-\mathcal{E}_{\text {global }}(t-\Delta t,-)}{\Delta t} \\
& =\text { dissipation }+ \text { input power } .
\end{aligned}
$$

In a model, if dissipation and forcing both vanish, $\mathcal{E}_{\text {global }}(t,-)=$ $\mathcal{E}_{\text {global }}(t-\Delta t,-)$, is a conservative quantity up to numerical accuracy. Thus, a reanalysis model run without databased adjustments should satisfy all of the conventional conservation requirements, including mechanical energy in the general sense of Eq. (4) with all sources and sinks accounted for.

In data assimilation as used in numerical weather forecasting and in most reanalyses, some observations of elements of $\mathbf{x}(t)$ are available at a specific time $t=\tau$, and the analyst seeks to take advantage of this new information to improve $\mathbf{x}(\tau,-)$ and hence $\mathcal{E}\left(\mathbf{r}_{i}, \tau,-\right)$, and $\mathcal{E}_{\text {global }}(\tau,-)$ and above all, the shortrange prediction, $\mathbf{x}(\tau+m \Delta t,-)$. Most such observations can be written generally as

$$
\mathbf{y}(\tau)=\mathbf{E}(\tau) \mathbf{x}(\tau)+\mathbf{n}(\tau),
$$

where the matrix $\mathbf{E}(\tau)$ selects one or more elements of the true $\mathbf{x}(\tau)$, a selection which can range from a single element $x_{i}(\tau)$ to a complicated weighted combination of any or all elements of $\mathbf{x}(\tau)$ (e.g., a regional volume-averaged temperature). All such measurements inevitably contain observational noise $\mathbf{n}(\tau)$, often characterized simply as a stochastic process of zeromean and covariance $\mathbf{R}(\tau)=\left\langle\mathbf{n}(\tau) \mathbf{n}(\tau)^{\mathrm{T}}\right\rangle$, with the single bracket implying a hypothetical expected value. Systematic errors, as always, demand separate, specific, treatment.

In most reanalyses, a predictor-corrector form of adjustment of $\mathbf{x}(\tau,-)$ is made in the form

$$
\tilde{\mathbf{x}}(\tau)=\tilde{\mathbf{x}}(\tau,-)+\mathbf{K}[\mathbf{y}(\tau)-\mathbf{E}(\tau) \tilde{\mathbf{x}}(\tau,-)] .
$$

Matrix $\mathbf{K}$ is a gain matrix that adjusts the forecast $\tilde{\mathbf{x}}(\tau,-)$ by an amount proportional to the misfit between the forecast of the data, and the data measurement, $\mathbf{y}(\tau)$ weighted inversely from their relative errors. In a rigorous derivation, with a number of assumptions being satisfied, $\mathbf{K}$ is the time-dependent (Kalman) gain matrix. (A bit more explanation of the structure is in appendix A). Despite the inference one might draw from the meteorological literature, true Kalman filters are almost never used because of computational loads and the requirement of formal statistical understanding of various fields and their errors. (Much of the formalism can be understood by resetting the time origin $\tau \rightarrow 0$, the last time data were used, with $\tilde{\mathbf{x}}(\tau)$ becoming the new initial condition $\tilde{\mathbf{x}}(\tau) \rightarrow \tilde{\mathbf{x}}(0)$ with new uncertainty $\mathbf{P}(\tau) \rightarrow \mathbf{P}(0)$. For present purposes, $t$ spans the entire time interval being considered).

In any case, however $\mathbf{K}$ is determined, the model calculated state, $\tilde{\mathbf{x}}(\tau,-)$, will jump to the modified state $\tilde{\mathbf{x}}(\tau)$. At that time, the transition from $\tilde{\mathbf{x}}(t=\tau,-)$ to $\tilde{\mathbf{x}}(t=\tau)$ no longer satisfies the model equation, and thus Eq. (4) fails: conservation rules do not apply at that time step. Energy or other property can jump at that time. With many observations distributed over any finite interval, and systematically used to adjust the state, overall computed energy can increase or decrease depending upon the 
nature of $\mathbf{K}$, the distributions of $\mathbf{E}\left(t_{n}\right), t_{n}=n \Delta t_{d}$, where $\Delta t_{d}$ is the interval between datasets, any systematic errors in the data, and the various time constants present in the model. A finding that energy increases can well be a physically correct inference, but the possibility of an artifact resulting from the insertion of observations must be accounted for. Similar considerations would apply to quantities such as mass or heat or any tracer variable.

\section{Energy conserving estimates: ECCO}

Failure of model physics (or chemistry or biology) when data are introduced via Kalman or other filters has been known and understood for decades. When state estimates are sought whose time evolution satisfies a known, conserving, system, the problem is labeled in the control literature as that of "smoothing" and which, also following that literature, is here called "state estimation." Thorough accounts of smoothing algorithms can be found in the textbooks already cited. Applications to oceanic systems are discussed in Wunsch (2006) and Heimbach et al. (2019) and other places.

The fundamental idea is to modify the data-adjusted estimate $\tilde{\mathbf{x}}(t)$ and the model, so that this third estimate [call it $\tilde{\mathbf{x}}(t,+)]$ satisfies a new (also adjusted) model over the entire time interval described by $t$ including all time steps when data were introduced. Without going into the details described in the references, model modification consists of systematically changing possibly all of the initial conditions, meteorological forcing conditions, model internal variables such as water depth, and numerous parameterization (including mixing) constants. Conservation rules apply once again. A two-sentence summary is that a nonlinear least squares system interpolation system is solved iteratively to minimize an objective function involving deviations from the observations and the prior estimates of initial and boundary conditions, etc. with the adjustable model being imposed through the method of Lagrange multipliers. The adjusted model is then run-and whose output satisfies both the data within error (mostly), adjustments within prior estimates of their errors (mostly), and conservation [Eq. (4)].

Here a direct estimate is made of mechanical energy over the 26-yr time interval 1992-2007 from the ECCO Consortium (see Fukumori et al. 2017, 2018, 2019, and references therein) state estimate known as version 4 release $4(\mathrm{v} 4 \mathrm{r} 4)$. This particular state estimate does not include any explicit form of surface, internalgravity, submesoscale, or mesoscale motions. Known evolution equations for energy (e.g., Olbers et al. 2012, p. 353+) involve interactions of all motions on all scales and are not simple. These fields are parameterized in various ways, e.g., through wind stress formulas and various dissipational algorithms of the MITgcm (Forget et al. 2015; Fukumori et al. 2017, 2018, 2019). Thus, in what follows, ocean turbulence closure formulas have been used in such a way that the explicit energetics of the missing motions are not accounted for, but internal parameterization values do change and contribute to calculated energies. ${ }^{3}$

\footnotetext{
${ }^{3}$ Higher-resolution ECCO state estimates do exist (Fenty et al. 2017; Heimbach et al. 2019), but span much shorter time intervals owing to the very heavy computational and human analysis costs involved.
}

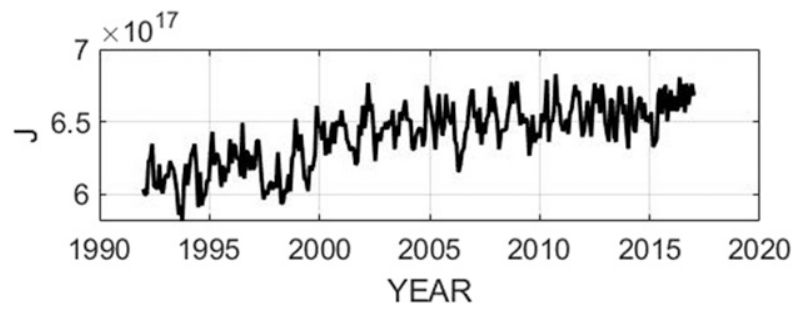

FIG. 2. Total potential energy (J) through time (monthly) of the sea surface from the state estimate values of $\eta$, including the part that is not available; $7 \times 10^{17} \mathrm{~J}$ is $0.7 \mathrm{EJ}$.

Here much of the same data are used as in H20, plus numerous other types, but only over the shorter interval where they infer a strong trend and when data become much denser. Although the observing system is never truly homogeneous over any extended interval, altimetric coverage becomes dense at the end of 1992, and the estimate used here is tightly tied to those datasets. These and many other data (Argo and CTD profiles, SST, SSS, gravity, atmospheric transfers, etc.) were used in the nonlinear least squares fit from 1992 to 2015 to the MITgcm. An explicit uncertainty (itself of sometimes uncertain accuracy) is attached to each data point, and the a posteriori misfits are known. ${ }^{4}$

No claim is being made that the state estimate or derived energies are "correct": no statistical procedure produces that. Results are best estimates based upon a variety of assumptions whose accuracy can be debated. They do at least provide an alternative interpretation of the data that might be considered seriously. Results are not definitive, and will remain so, until either the remaining energy reservoirs are resolvable with numerical skill, or the parameterizations (closures) are better understood. The "ECCO-ocean" is not the real ocean, but it is a useful surrogate.

\section{a. Surface potential energy}

What follows is focused upon the ECCO surface ocean, with some full water depth results described briefly at the end. All results are from a conservative system (up to the MITgcm numerical errors). The near-surface ocean has by far the densest direct observations. Consider the PE of the total surface elevation $\eta(\phi, \lambda, t)$ (a subset of the state vector) written as

$$
\mathrm{PE}_{\eta}(\phi, \lambda, t)=\mathrm{PE}_{\eta}\left(\mathbf{r}_{j}, t_{k}\right)=\frac{1}{2} g \rho_{0} \eta\left(\mathbf{r}_{j}, t_{k}\right)^{2}, 0 \leq t \leq \tau \leq T_{\mathrm{dur}},
$$

\footnotetext{
${ }^{4}$ The widely recognized remaining major shortcoming of $\mathrm{v} 4 \mathrm{r} 4$ is the absence, because of computational loads, of the complete error estimates of the resulting state $\tilde{\mathbf{x}}(t,+)$ and of the various adjusted fields. Consequently, this paper makes no claims to statistical significance. Kalmikov and Heimbach (2014) have discussed an approach through inverse Hessians, and Heimbach et al. (2011) have described related sensitivities determined from the Lagrange multipliers (sometimes called the "dual" or "adjoint" model).
} 
TABLE 3. Various potential energy levels associated with different combinations of elements of $\eta$ from ECCOv4r4. Double brackets denote a global ocean integral; $\eta$ subscripts are used to denote surface contributions only.

\begin{tabular}{lll}
\hline \hline & $\mathrm{EJ}\left(10^{18} \mathrm{~J}\right)$ equivalent & \multicolumn{1}{c}{ Comment } \\
\hline $\mathrm{PE}_{\eta}$ element integrals & & \\
$\left\langle\left\langle\eta^{2}\right\rangle\right\rangle$ & 0.6 & Mean square $\eta$ 26-yr monthly values \\
$\left\langle\left\langle\eta(2017)^{2}-\eta(1992)^{2}\right\rangle\right\rangle$ & 0.05 & Change in total last to first year (see Fig. 2) \\
$\left\langle\left\langle\langle\bar{\eta}\rangle^{2}\right\rangle\right\rangle$ & 0.04 & 26-yr time-mean, global-mean elevation \\
$\left\langle\left\langle\left[(\bar{\eta}-\langle\bar{\eta}\rangle) / A_{0}\right]^{2}\right\rangle\right\rangle$ & 0.3 & Deviation of time mean from its spatial mean \\
$\left\langle\left\langle(\eta-\langle\eta\rangle)^{2}\right\rangle\right\rangle$ & 0.007 & Deviation of monthly values from time and space mean \\
$\mathrm{KE}_{\eta}$ element integrals & & \\
Surface time mean $(10-\mathrm{m}$ depth) & 0.38 & \\
Surface geostrophic from $\bar{\eta}$ & 0.0002 & \\
\hline
\end{tabular}

where gravity $g$ and $\rho_{0}$ are taken as constant and constitute an acceptable approximation. The terms $\phi$ and $\lambda$ are latitude and longitude and time intervals $\tau \leq T_{\text {dur }}$, the total duration of values. (Context will make clear that in much of what follows: "ocean" must be understood to be the ECCO ocean.) Position vector $\mathbf{r}_{j}$ and time $t_{k}$ are introduced to represent the discrete nature of the available values. Potential energy is not a direct measure of the speed of the ocean circulation, but is intimately related to it because kinetic energy features always have some associated potential energy, and which, depending on details, can dominate the energetics (Gill et al. 1974). PE also includes the effects of changes in buoyancy forcing to and from the atmosphere. As a Boussinesq model, $\eta$ includes the so-called Greatbatch correction (Griffies 2004) for the volume effects of steric changes.

If integrated over the ocean,

$$
\left\langle\left\langle\mathrm{PE}_{\eta}\left(t_{k}\right)\right\rangle\right\rangle=\frac{1}{2} g \rho_{0} \iint_{A_{0}} d S \eta\left(\mathbf{r}_{j}, t_{k}\right)^{2} \approx \frac{1}{2} g \rho_{0} \sum_{j} \eta\left(\mathbf{r}_{j}, t_{k}\right)^{2} \Delta S_{j},
$$

where $\Delta S_{j}$ is the area represented by $\eta\left(\mathbf{r}_{j}, t_{k}\right)$. (The double angle bracket again denotes a global ocean integral). In the real ocean, $\eta$ is made up of a great variety of processes ranging from ordinary water waves, near-surface turbulence properties, tides, submesoscale and mesoscale eddies, and the larger-scale circulation, perhaps "quasigeostrophic." Only the last element is represented explicitly in the state estimate after averaging to months or years. Not accounted for here is the potential energy contained in floating sea ice: melting sea ice transfers that energy into both kinetic and potential energies of the fluid ocean.

Over any fixed time-averaging interval $\tau$ (redefining $\tau$ ), the individual quadratic contribution of the various processes available in the state estimate can be calculated. The $\mathrm{PE}_{\eta}$ of the present total $\left\langle\left\langle\mathrm{PE}_{\eta}\left(t_{k}\right)\right\rangle\right\rangle$, including the nonavailable $\mathrm{PE}_{\eta}$ from the spatial average each month, as a function of time, is shown in Fig. 2. Although an increase over the first half of the interval to about 2005 exists, nothing like an overall linear trend appears and average values are stable after that year. (Recall that the H20 estimated energy increase is $2 \times 10^{17} \mathrm{~J}$ ).

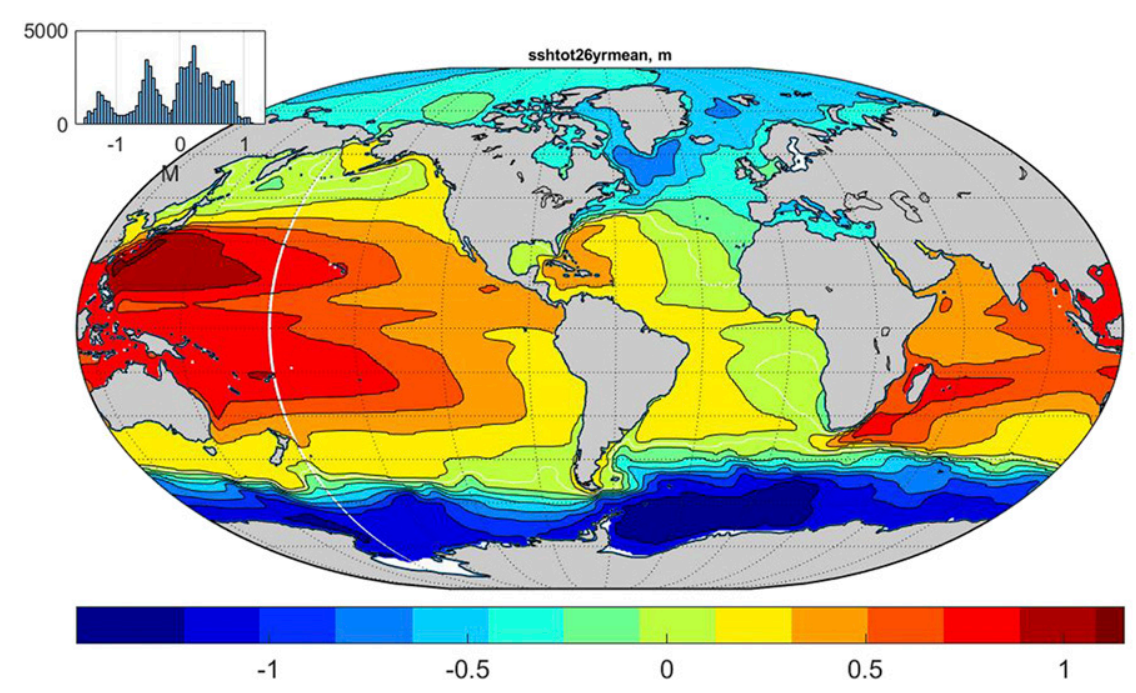

FIG. 3. The 26-yr time average of the sea surface elevation in the ECCO v4r4 estimate (m). Compare Fig. 14 in Fukumori et al. (2018) for v4r3 (20 years). Note the strongly multimodal character (inset) of the values such that a conventional variance is not very meaningful. 


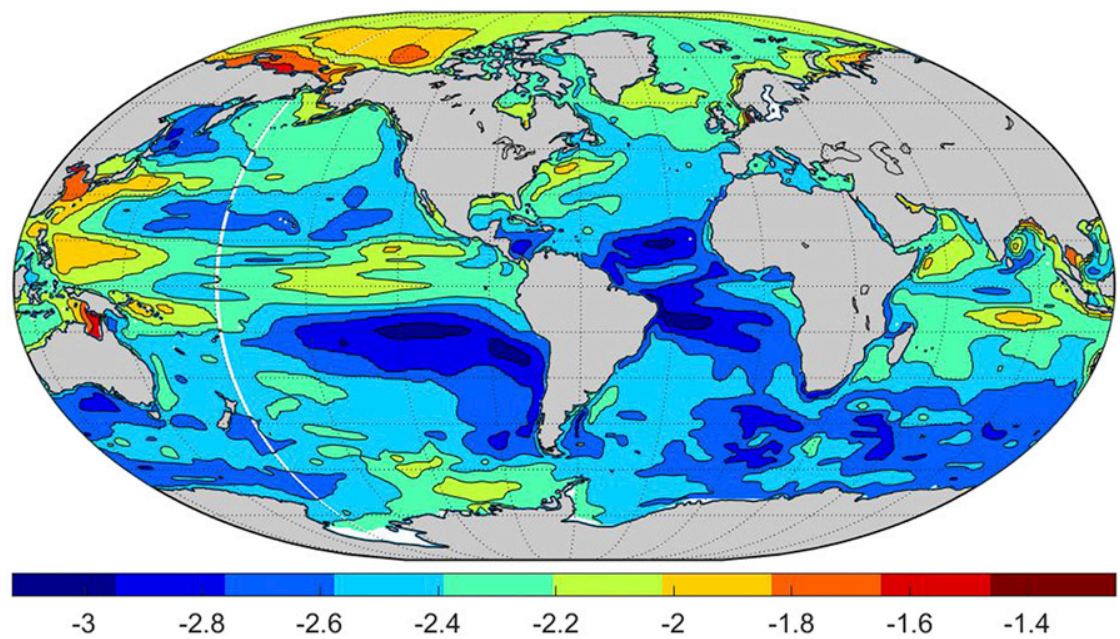

FIG. 4. Logarithm of the monthly variance of $\eta\left(\mathrm{m}^{2}\right)$ through time (26 years) depicting the spatial variability and hence part of the ocean spatial sampling problem.

Write $\eta$ as

$$
\begin{aligned}
\eta(\phi, \lambda, t) & =\eta\left(\mathbf{r}_{j}, t_{k}\right)=\bar{\eta}\left(\mathbf{r}_{j}, \tau\right)+\eta^{\prime}\left(\mathbf{r}_{j}, t_{k}, \tau\right)+\eta_{\mathrm{un}}^{\prime}\left(\mathbf{r}_{j}, t_{k}, \tau\right) \\
& =\bar{\eta}\left(\mathbf{r}_{j}, \tau\right)+\left\langle\eta^{\prime}\left(\mathbf{r}_{j}, t_{k}, \tau\right)\right\rangle+\eta_{1}^{\prime}\left(\mathbf{r}_{j}, t_{k}, \tau\right)+\eta_{\mathrm{un}}^{\prime}\left(\mathbf{r}_{j}, t_{k}, \tau\right),
\end{aligned}
$$

where the overbar is a finite averaging interval over $\tau \leq$ $T_{\text {dur }}$-but possibly much less ( $1 \mathrm{~s}, 1 \mathrm{~h}, 1$ day, 1 month, etc.). Single angle brackets $\langle$.$\rangle denote a spatial average, not the$ integral, over the entire ocean. The term $\eta_{\text {un }}^{\prime}$ is everything at high frequency and/or high wavenumber that is omitted here. Table 3 lists some of the basic integrals of surface energies.

Ideally (see Davis 1994) quantities such as that of the energy of the time average over intervals $\tau$ should be uncorrelated with properties of the energy anomalies in that same interval. Absent any known frequency spectral gap, possibilities exist for physical misinterpretation of the separated fields. Insight into the physics of change requires separating spatially constant contributions in both $\bar{\eta}\left(\mathbf{r}_{j}, \tau\right),\left\langle\eta^{\prime}\left(\mathbf{r}_{j}, t_{k}, \tau\right)\right\rangle$, from both the spatially variable parts, and the time dependencies. In general, the spatial mean $\left\langle\eta^{\prime}\left(\mathbf{r}_{j}, t_{k}, \tau\right)\right\rangle$ does not vanish, e.g., owing to a globally uniform rise or fall in sea level. Because such a movement does involve a real change in potential energy, it must be accounted for, and hence a second anomaly $\eta_{1}^{\prime}$ relative to the spatial and time average elevation is introduced into Eq. (9). The spatial mean $\eta_{1}^{\prime}\left(\mathbf{r}_{j}, t_{k}, \tau\right)$ is the only element that has both time and space averages or integrals that vanish.

The surface elevation $\eta$ at any time is, in principle, measured relative to an idealized underlying surface, $r_{E}(\phi$, $\lambda)=N_{G}(\phi, \lambda)+S_{p}(\phi, \lambda)$, measured from a reference
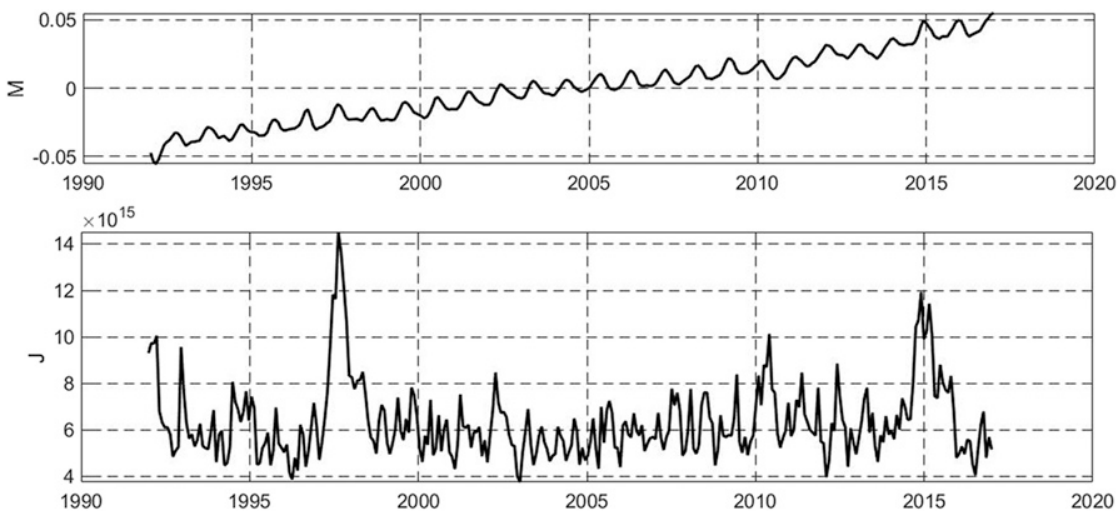

FIG. 5. (top) Global area-weighted spatial average of $\eta^{\prime}\left(\mathbf{r}_{j}, t\right)$ by month, $\left\langle\left\langle\eta^{\prime}\left(\mathbf{r}_{j}, t\right)\right\rangle\right\rangle / A_{0}$, showing the general rise in the sea level anomaly owing to water addition and heating. (The overall time mean is zero by subtraction of the time mean $\bar{\eta}$.) The associated PE, about $0.2 \mathrm{EJ}$, would not generally be available for conversion into KE. (bottom) The total PE by month, with the monthly global space average removed. The spatial distribution of the average rise strongly affects the PE estimate. ENSO 1997/98 and 2014-16 cycles show as peaks. 
ellipsoid $S_{p}(\phi, \lambda)$ and known as the "geoid" $N_{G}(\phi, \lambda)$. Historically, it was defined as the gravitational equipotential to which the ocean surface would conform if it were brought to complete rest without forcing and the density rendered uniform (in some definition). ${ }^{5}$ In practice, it is today measured by gravity satellites and a best estimate $\tilde{N}_{G}\left(\mathbf{r}_{j}\right)$ is chosen somewhere near "mean" sea level. Parameter $r_{E}$ varies relative to the center of Earth by order $26 \mathrm{~km}$, and with superimposed undulations of order $100 \mathrm{~m}$. It thus also becomes a reference surface for the state estimate (see, e.g., Chambers et al. 2018). ${ }^{6}$ Whichever is specifically chosen, a uniform radius change $\Delta \eta_{0}$ as might occur if the ocean were warmed or cooled overall or had water added or subtracted, would generate an apparent surface $\mathrm{PE}_{\eta}$ change of

$$
\left\langle\left\langle\Delta \mathrm{PE}_{\eta}\right\rangle\right\rangle \approx \frac{1}{2} g \rho_{0} S_{0}\left(\Delta \eta_{0}\right)^{2}
$$

with $S_{0}=\sum_{j} \Delta S_{j}$ being the total ocean area. The term $\left\langle\left\langle\Delta \mathrm{PE}_{\eta}\right\rangle\right\rangle$ would represent a change in the total potential energy of the ocean-the latter being a vast number $\left(\approx 2 \times 10^{25} \mathrm{~J}, 20\right.$ yottajoules; see Table 1) - and which would not be "available" to the ocean circulation except on geological time scales. For a 1m uniform offset from $\tilde{N}_{G},\left\langle\left\langle\Delta \mathrm{PE}_{\eta}\right\rangle\right\rangle \approx 2.6 \times 10^{18} \mathrm{~J}$, and which is of interest only if the surface circulation were to be modified on a very long time scale.

Consider now the time mean $\bar{\eta}\left(\mathbf{r}_{j}\right)$ over $\tau=T_{\text {dur }}=26 \mathrm{yr}$ displayed in Fig. 3 relative to the underlying estimated geoid surface. This field reproduces the conventional elements of the large-scale surface ocean circulation, including the boundary currents, the gyres, the Antarctic Circumpolar Current, etc. Surface potential energy of the time-mean circulation is

$$
\overline{\mathrm{PE}_{\eta}}\left(\mathbf{r}_{j}, T_{\mathrm{dur}}\right)=\frac{1}{2} g \rho_{0} \bar{\eta}\left(\mathbf{r}_{j}, T_{\mathrm{dur}}\right)^{2} .
$$

Its space average $\left\langle\bar{\eta}\left(\mathbf{r}_{j}, T_{\text {dur }}\right)\right\rangle=0.15 \mathrm{~m}$, is indistinguishable from a uniform geoid offset and so is unavailable potential energy of $\approx 5.8 \times 10^{16} \mathrm{~J}$. Spatial variance of this field about its mean corresponds to about $(0.4 \mathrm{~m})^{2}$ for an energy of $\approx 4 \times$ $10^{17} \mathrm{~J}$ (but note the very non-Gaussian distribution of values in $\bar{\eta}$ shown in Fig. 3). This time-mean spatial variability is supported by time-mean wind structures, mean thermohaline

\footnotetext{
${ }^{5}$ The notion of "mean sea level" for an unforced, constant density ocean, as derived from actual observations, is no longer a scientifically practical definition of the geoid. Discussion can be confined to adoption of some convenient geopotential as inferred from space-borne measurements. Oceanographically, a spatially uniform mean offset appears only in the potential energy equation, with flow fields being sensitive only to the slope of the actual sea surface relative to a geopotential.

${ }^{6}$ Omitting discussion of time dependence in $N_{G}(\phi, \lambda, t)$ from mass redistributions of all sorts, including tides and their selfattraction, glacial isostatic adjustment, and changes in the mass distribution of the ocean circulation itself. Potential energy tied up in floating sea ice is being ignored as it covers a relatively small area. Because of the underlying time dependence, ECCOv4r4 uses a geoid that might be labeled as from the "epoch" year 2010.
}

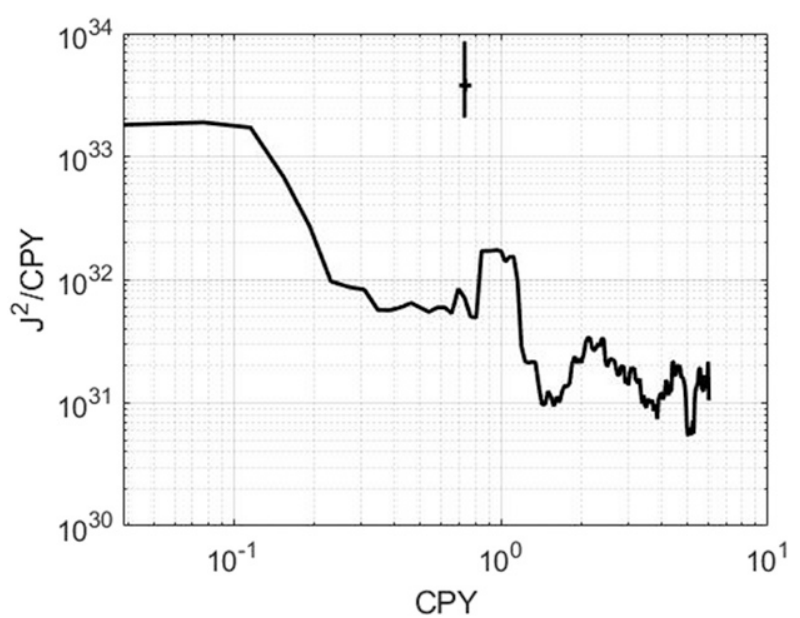

FIG. 6. Power density of the total (available and not available) potential energy through time with a linear trend removed. An annual peak is conspicuous. The $95 \%$ confidence interval is highly approximate based upon the assumption that the time series is Gaussian.

exchanges, and time-mean nonlinearities of the parameterized higher-wavenumber variability. The extent to which this energy could be available for conversion to kinetic energy would be highly time-scale dependent (e.g., in theory, suddenly, or very slowly, releasing a spatially structured time-mean inverted barometer component would produce very different responses).

Superimposed upon this field at any given time is a perturbation field $\eta^{\prime}(\phi, \lambda, t)$, where time $t$ in theory changes over intervals $\Delta t$ running from seconds or less to the age of the ocean. The logarithm of its 26-yr average spatial variance at each point is shown in Fig. 4. Large values are generally associated with energetic western boundaries, a complicated tropical dependence, and a limited region in the Southern Ocean. Kinetic energy and the surface potential energy are quadratic in the state vector, and care must be taken in computing energies over finite time intervals shorter than 26 years, by separating spatial averages $\langle\eta\rangle$ at any given time, and which are unavailable over particular intervals. Apart from the unavailable surface PE, no obvious trend exists in the surface potential energy over this $26-y$ r period.

The global spatial average of the anomaly $\left\langle\eta^{\prime}\right\rangle$ relative to the 26yr mean, is displayed in Fig. 5. A general expected rise in mean sea level is seen, of about $10 \mathrm{~cm}$ over 26 years, $\approx 3.8 \mathrm{~mm} \mathrm{yr}^{-1}$ (see Wunsch 2016; Fukumori et al. 2019) plus an annual cycle. The associated unavailable $\mathrm{PE}_{\eta}$ is a tiny contribution to the general circulation total. Its spectral density estimate is shown in Fig. 6. An annual peak, a weak overtone and a tendency to low-frequency white noise are seen. The annual peak is sufficiently energetic that it enters the discussion of sampling densities to avoid aliasing.

\section{b. The ECCO ocean surface $K E_{\eta}$}

To provide another reference point, as listed in Table 1, a barotropic velocity (uniform top to bottom) of $1 \mathrm{~cm} \mathrm{~s}^{-1}$ corresponds to about $0.7 \mathrm{EJ}$; see Table 1 ; if confined to the top 


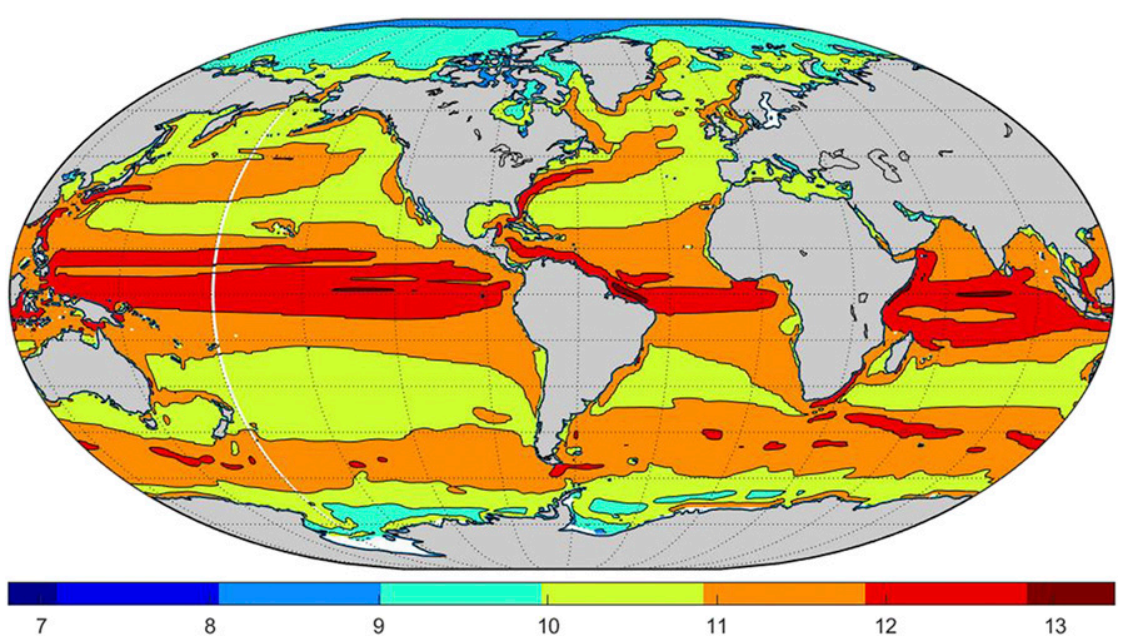

FIG. 7. Log of the time average surface kinetic energy over 26 years. Western boundaries, the tropics, and the Southern Ocean are also conspicuously high as expected.

$10 \mathrm{~m}$ it is $2 \times 10^{15} \mathrm{~J}$. The time average of the sea surface $\mathrm{KE}_{\eta}$ (top $10 \mathrm{~m}$ ) is displayed in Fig. 7, and its globally integrated variance value through time is in Fig. 8. Global RMS (from monthly values) surface speed is $\approx 4 \mathrm{~cm} \mathrm{~s}^{-1}$. Visually, a weak quasi-linear trend in time appears, but the degree of variability does increase as the data become more dense, and whether that is a coincidence is not known. A robust slope estimator (the Theil-Sen median; variables are significantly non-Gaussian) is $1.9 \times 10^{14} \mathrm{~J} \mathrm{yr}^{-1}$ or a speed increase of about $1 \mathrm{~cm} \mathrm{~s}^{-1} \mathrm{yr}^{-1}$ if confined to the top $10 \mathrm{~m}$ and about $0.3 \mathrm{~cm} \mathrm{~s}^{-1} \mathrm{yr}^{-1}$ if the whole water column is involved. [This estimator was used also by Young and Ribal (2019).]

By running a 400-member bootstrap sample ensemble (Wilcox 2010) the distribution displayed in Fig. 8 is obtained with a standard deviation of $\pm 5.3 \times 10^{13} \mathrm{~J} \mathrm{yr}^{-1}$, corresponding to a speed increase then of about $1 \pm 0.5 \mathrm{~cm} \mathrm{~s}^{-1} \mathrm{yr}^{-1}$ (top $10 \mathrm{~m}$ only). The calculated slope would be marginally positive at two standard deviations, and thus a weak increase in surface $\mathrm{KE}_{\eta}$ appears where "weak" is relative to the estimated background values in Tables 1 and 2 and Fig. 1. (The reader is strongly cautioned, as in a previous footnote, that time-dependent errors in the state estimate itself are not accounted for, only variability in the calculated $\mathrm{KE}$ ).

\section{GEOSTROPHY}

In discussions of the general circulation at the sea surface, focus is commonly on the geostrophic component. In practice, this component is expected to be small compared to the flow field at the surface owing to the presence of a very large variety of processes ranging from surface gravity waves through boundary layer flows to mesoscale and submesoscale contributions. Geostrophic flow kinetic energy is discussed in appendix B and no obvious trend is seen.

\section{c. Water column total kinetic energy}

A discussion of the time history of kinetic plus potential energy with depth deserves a detailed paper of its own: the depth-mean spatial variability of the various state estimate fields is very large (Wunsch 2020) with a dependence upon topographic features. Discussion of PE that is available becomes complicated (Roquet 2013, among many others). For context, however, in the present discussion, Fig. 9 shows the vertical integral over three depth ranges of globally integrated KE in the state estimate. No major trend has occurred over the record length. A weak increase to 2005 and then a slight decrease again, in the total, might be inferred. The reader is reminded that high-wavenumber or high-frequency components (shorter than 1 month) are not included.

\section{Summary and discussion}

$\mathrm{H} 20$ have made the interesting and provocative suggestion that the ocean circulation has been "speeding up" over the past

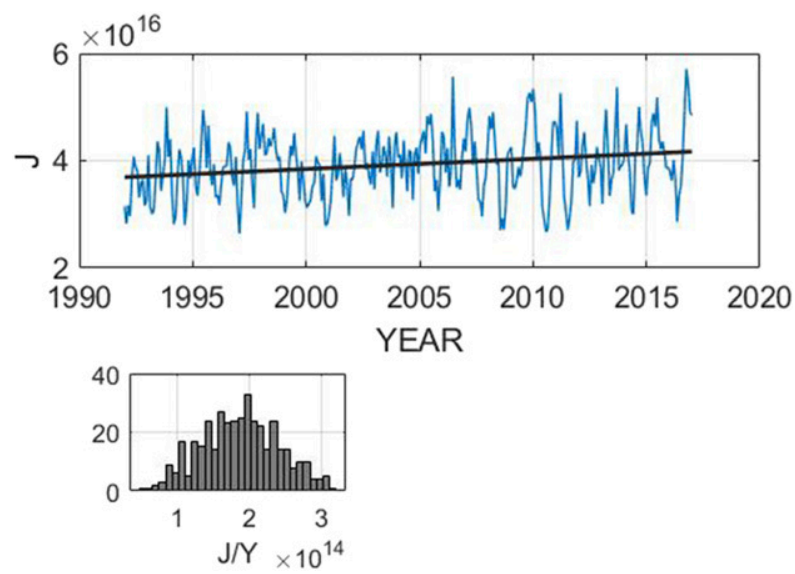

FIG. 8. (top) Top-layer kinetic energy through time in joules. Also shown is the Theil-Sen (median) slope. (bottom) A 400member bootstrap resampling was used to generate the histogram values, which would imply a significant weak trend. The reader is reminded however, that state estimate error is not included-merely the slope of the samples generated from it. 


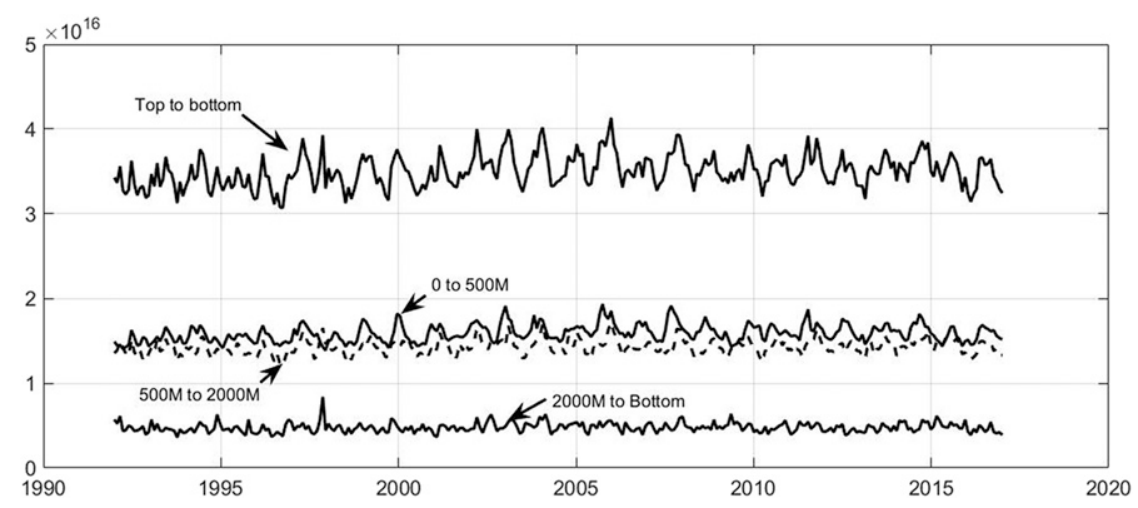

FIG. 9. Kinetic energy (J) with time integrated between the near-surface and $500 \mathrm{~m}$, from 500 to $2000 \mathrm{~m}$, and from $2000 \mathrm{~m}$ to the bottom. These changes necessarily arise from the changes in the control vector (atmospheric exchanges, internal conversions from parameters, etc.), but that discussion is beyond the present intended scope.

several decades. Such a change has implications for numerous climate-related properties such as heat and nutrient transports. Neither water waves nor estimated wind stress amplitudes appear to have changed very much over the last decades and while not necessarily in conflict with the $\mathrm{H} 20$ results, do not produce obvious support. See Young and Ribal (2019), who used a comparatively homogeneous satellite dataset, but technical questions and some controversy do remain.

Because of its importance and interest, the question of trends in the general circulation is further reexamined here from two points of view: 1) the magnitudes of existing reservoirs of quasi-steady mechanical energy and of their sources and sinks and 2) from an energy-conserving state estimate.

The first approach offers an essentially qualitative, plausible conclusion: as seen in Tables 1 and 2 and Fig. 1, if the ocean is assumed to be in a quasi-steady energy state, published implied changes are small fractions of the estimated resident energy. In most cases, a 1\% fluctuation or error in those background states would swamp any proposed trend in their values. The reader can judge whether $1 \%$ accuracies reflect existing oceanographic knowledge. (Precision would instead be of main relevance if the observing system and deployments were homogeneous in time.)

For the second approach, it is first noted that "reanalyses" do not conserve physical properties and their energetics are susceptible to trends in observing systems types, numbers, and distributions (see particularly Bengtsson et al. 2004). For that reason the global surface and deeper circulations are evaluated using the 26-yr long monthly ECCO version 4 release 4 estimates. These estimates derive from solutions of a free-running version of the MITgcm, satisfying mechanical energy budgets up to numerical issues.

An estimated trend in sea level (about $3.5 \mathrm{~mm} \mathrm{yr}^{-1}$ ) gives rise to a small contribution to the unavailable potential energy of the general circulation. Surface energy results are otherwise dominated by the potential energy of the spatial surface variations [as one infers from Gill et al. (1974)]. Considerable fluctuations are found but no evidence is seen of any notable trend. A weak trend of $\approx(1.9 \pm 0.5) \times 10^{14} \mathrm{~J} \mathrm{yr}^{-1}$ appears in total surface kinetic energy corresponding to a surface (top
$10 \mathrm{~m}$ ) speed increase of about $0.9 \pm 0.45 \mathrm{~cm} \mathrm{~s}^{-1} \mathrm{yr}^{-1}$ (one standard deviation of the ECCO estimates). Preliminary estimates of full-water-column KE do not show any obvious trends. Energies of the full water column require a discussion of the fraction that is available PE, and which is more complicated to calculate than is KE, as discussed in the references.

The inference that the ocean has "sped up" slightly, with a corresponding increase in energy levels, remains possible. A more definite conclusion requires better understanding of the influences on accuracy of changing technologies and data distributions. The goal of a fully adequate ocean observing system, maintained for decades into the future, is the most straightforward approach. "Fully adequate" requires specific knowledge both of required and achievable accuracy. Energy conserving model-based systems accounting for the dominant energy reservoirs at the required accuracy for findings of change are also needed and perhaps will be forthcoming. Many of these requirements are fully generic-extending to determining global changes in numerous properties such as salt or carbon content.

Acknowledgments. Supported by the ECCO Consortium funding, but written and revised at home in the Trump apocalypse shutdown. Diana Spiegel helped greatly with the file handling. I thank Nicolas Rascle and Fabrice Ardhuin for helpful comments on the surface gravity wave field and Patrick Heimbach, Fabien Roquet, Detlef Stammer, and an anonymous reviewer for useful suggestions. The entire ECCO consortium membership made this work possible.

Data availability statement. The entire state estimate output is available through the Jet Propulsion Laboratory. See https:// www.ecco-group.org/docs/v4r4_synopsis.pdf.

\section{APPENDIX A}

\section{Filters}

In the predictor-corrector form of reanalyses, or in the true Kalman filter, the estimated state variables $\tilde{\mathbf{x}}(t)$ made at time $t$ 


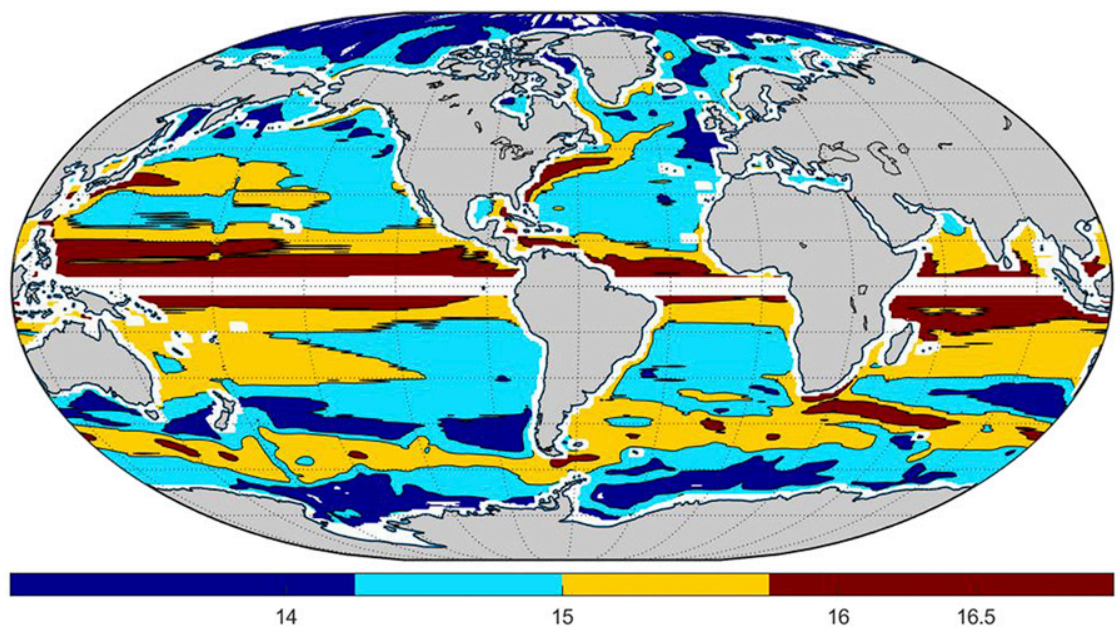

FIG. B1. Logarithm of the 26-yr average geostrophic kinetic energy (J) from $\bar{\eta}(\phi, \lambda)$. Owing to a tendency to producing long zonal streaks, this field has been smoothed over $5 \times 5$ grid points before contouring. Compare to the map of total surface KE.

from some initial time (which might be only one time step $\Delta t$ in the past, or more commonly many such time steps in the past) an adjustment is made as

$$
\tilde{\mathbf{x}}(t)=\tilde{\mathbf{x}}(t,-)+\mathbf{K}_{g}(t)[\mathbf{y}(t)-\mathbf{E}(t) \tilde{\mathbf{x}}(t,-)]
$$

The convention is used that the minus sign in the argument denotes an estimate made by the model alone, at that time. It is dropped after data are incorporated into the estimate. In a true Kalman filter,

$$
\begin{aligned}
\mathbf{K}_{g}(t) & =\mathbf{P}(t,-) \mathbf{E}(t)^{\mathrm{T}}\left[\mathbf{E}(t) \mathbf{P}(t,-) \mathbf{E}(t)^{\mathrm{T}}+\mathbf{R}(t)\right]^{-1}, \\
\mathbf{P}(t,-) & =\left\langle[\tilde{\mathbf{x}}(t,-)-\mathbf{x}(t)][\tilde{\mathbf{x}}(t,-)-\mathbf{x}(t)]^{\mathrm{T}}\right\rangle,
\end{aligned}
$$

noting that $\mathbf{P}(t,-)$ is the expected "prediction error" covariance matrix from the prediction model alone. Here the bracket denotes expected value). The matrix $\mathbf{R}(t)$ represents the zeromean (assumed) observational noise. In most reanalyses as in weather forecasting, $\mathbf{K}_{g}$ is usually taken as constant through time because computation of the covariance matrices $\mathbf{P}(t,-)$ and then $\mathbf{P}(t)$, after data are used, each requires running the model $2 N$ times at each time step where $N$ is the dimension of the state vector, an impractical computation with modern GCM state vectors. ${ }^{\mathrm{A} 1}$

Starting from any time $t=0$, the prediction model produces a state vector estimate at time $\tau, \tilde{\mathbf{x}}(\tau,-)$ satisfying the energy rule

\footnotetext{
${ }^{\text {A1 }}$ The inability to compute the covariance matrices through the Kalman filter formulation is sometimes addressed by generating an ensemble of solutions and calculating the variances from the ensemble members (e.g., Evensen 2009). In turn, however, a heavy burden is placed on the sensible choice of a probability density for the ensemble members, and typically ensemble sizes are so much smaller than the state estimate dimension $N$ that the resulting covariances would be highly singular (rank deficient).
}

Eq. (4) [here quadratic in elements of $\tilde{\mathbf{x}}(\tau,-)$ ] for the whole time period $0 \leq t \leq \tau$ as long as no data have been introduced. If $\tilde{\mathbf{x}}(\tau,-)$ in Eq. (1) is replaced by $\tilde{\mathbf{x}}(\tau)$, the adjusted state vector no longer conforms to Eq. (4). One can reset $t=0$, at $t=\tau$, and energy conservation is obtained until such time as more data are introduced after some time steps. Over a finite interval, with many data insertion points, the apparent $\varepsilon(t)$ can have increased, decreased, or stayed the same, depending upon a large number of issues, including the structure of the observation matrix $\mathbf{E}(t)$ the time constants of the model, the relative errors of the "known" forcing used, and the assumed covariances.

To assure the availability of a state estimate that does satisfy energy and other conservation rules, one generates yet another estimate, $\tilde{\mathbf{x}}(t,+)$, for any time $t$, that also uses all of the data formally future to $t$ in what is known as a "smoothing" algorithm for which several numerical algorithms exist. As used in ECCO (see the various references given above), the algorithm is based upon an iterative least squares method with the adjusted model enforced through the use of Lagrange multipliers. In practice, and ignored in this paper, nonlinearity of the model leads to iterative linearization, but the basic ideas remain unchanged.

\section{APPENDIX B}

\section{Surface Geostrophic KE}

The state estimate contains a parameterization of the surface turbulent boundary layer, but does not resolve the mesoscale or submesocale (although these too, are parameterized; see Fukumori et al. 2017, 2018). Nonetheless, a comparison can be made between the apparent geostrophic kinetic energies, and the total arising from the model near-surface turbulence closure scheme. In general (Gill et al. 1974, their appendix) in baroclinic quasigeostrophic flows, potential energy is far larger than kinetic.

Consider the apparent geostrophic velocity at the sea surface, 


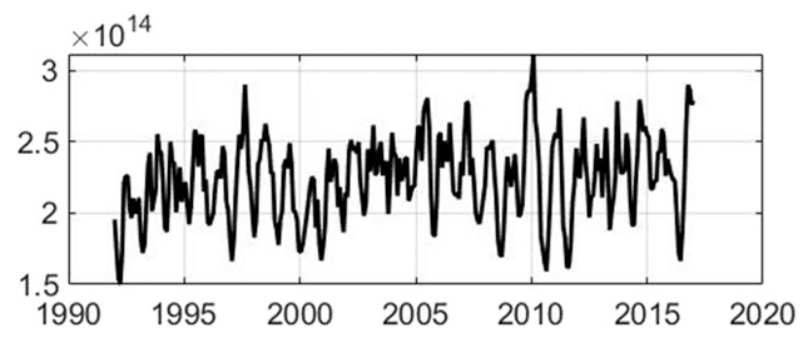

FIG. B2. Monthly time series of total geostrophic surface kinetic energy through time. Mean is $\approx 2.2 \times 10^{14} \mathrm{~J}$. An El Niño occurred in 1997/98, which shows a peak, and in 2014-16, where there is no peak. No explanation is offered here for the 2010 extreme.

$$
\begin{aligned}
& u_{g}(\phi, \lambda, t) \approx-\frac{g}{f(\phi) a} \frac{\partial \eta}{\partial \phi}, \eta=\bar{\eta}+\eta^{\prime}, \\
& v_{g}(\phi, \lambda, t) \approx \frac{g}{f(\phi) a \cos \phi} \frac{\partial \eta}{\partial \lambda},
\end{aligned}
$$

where $a$ is a radius of Earth. Justification in textbooks for oceanic geostrophic balance is normally written for an "instantaneous" ocean-one averaged over a few inertial periods. The extent to which geostrophic balance applies accurately to a $26-y r$ or monthly time average of hourly values on distances of order $1^{\circ}$ horizontally remains uncertain owing to the finite time-average nonlinear terms.

Taking the result at face value, however, the surface geostrophic KE at a point is now defined as

$$
\mathrm{KE}\left(\mathbf{r}_{j} . t\right)=\frac{g^{2} \rho_{0}}{f(\phi)^{2} a^{2}}\left[\left(\frac{\partial \eta}{\partial \phi}\right)^{2}+\frac{1}{\cos ^{2} \phi}\left(\frac{\partial \eta}{\partial \lambda}\right)^{2}\right],
$$

where $\eta$ is again broken into the temporal mean and deviation.

For reference, if $\bar{\eta}$ varies globally by $1 \mathrm{~m}$ over distances of $1000 \mathrm{~km}$, and taking the value of $f\left(30^{\circ}\right)$, the kinetic energy of the mean geostrophic flow $\operatorname{KE}\left(T_{w}\right) \approx 1.5 \times 10^{15} \mathrm{~J}$ and which is small compared to the mean surface PE, or the total eddy estimate of order $10^{19} \mathrm{~J}$ (over the full water column). Values within $2^{\circ}$ of the equator are omitted. Figure B1 displays the 26-yr average total geostrophic energy. Tropical regions, where geostrophy becomes susceptible to noise, western boundary currents, and major parts of the Southern Ocean are the major features. Spatial inhomogeneity with its consequent sampling difficulties, are evident.

Total KE of the time mean surface geostrophic flow is $2.2 \times$ $10^{14} \mathrm{~J}$. Figure B2 displays the global sum monthly over the 26 years. No monotonic change is visible. Indications of the ENSO episodes can be seen, however.

\section{REFERENCES}

Abdalla, S., and P. A. E. M. Janssen, 2018: Monitoring waves and surface winds by satellite altimetry: Applications. Satellite Altimetry over Oceans and Land Surfaces, D. Stammer and A. Cazenave, Eds., CRC Press, 381-426.

Bengtsson, L., S. Hagemann, and K. I. Hodges, 2004: Can climate trends be calculated from reanalysis data? J. Geophys. Res., 109, D11111, https://doi.org/10.1029/2004JD004536.

Chambers, D., O. B. Andersen, S. Bettadpur, M. H. Rio, R. Rummel, and D. Wiese, 2018: Auxiliary space-based systems for interpreting satellite altimetry, satellite gravity. Satellite Altimetry Over Oceans and Land Surfaces, D. Stammer and A. Cazenave, Eds., CRC Press, 149-186.

Davies, J. H., and D. R. Davies, 2010: Earth's surface heat flux. Solid Earth, 1, 5-24, https://doi.org/10.5194/se-1-5-2010.

Davis, R. E., 1994: Diapycnal mixing in the ocean: Equations for largescale budgets. J. Phys. Oceanogr., 24, 777-800, https://doi.org/ 10.1175/1520-0485(1994)024<0777:DMITOE > 2.0.CO;2.

Dee, D. P., and Coauthors, 2011: The ERA-Interim reanalysis: Configuration and performance of the data assimilation system. Quart. J. Roy. Meteor. Soc., 137, 553-597, https://doi.org/ 10.1002/qj.828.

de Verdière, A. C., T. Huck, S. Pogossian, and M. Ollitrault, 2018: Available potential energy in density coordinates. J. Phys. Oceanogr., 48, 1867-1883, https://doi.org/10.1175/JPO-D-170272.1.

Dewar, W. K., R. J. Bingham, R. L. Iverson, D. P. Nowacek, L. C. St. Laurent, and P. H. Wiebe, 2006: Does the marine biosphere mix the ocean? J. Mar. Res., 64, 541-561, https://doi.org/10.1357/ 002224006778715720.

Evensen, G., 2009: Data Assimilation: The Ensemble Kalman Filter. Springer Verlag, $332 \mathrm{pp}$.

Fenty, I., D. Menemenlis, and H. Zhang, 2017: Global coupled sea ice-ocean state estimation. Climate Dyn., 49, 931-956, https:// doi.org/10.1007/s00382-015-2796-6.

Ferrari, R., and C. Wunsch, 2009: Ocean circulation kinetic energy: Reservoirs, sources, and sinks. Annu. Rev. Fluid Mech., 41, 253282, https://doi.org/10.1146/annurev.fluid.40.111406.102139.

$\longrightarrow$, and - 2010: The distribution of eddy kinetic and potential energies in the global ocean. Tellus, 62A, 92-108, https:// doi.org/10.3402/tellusa.v62i2.15680.

Forget, G., J.-M. Campin, P. Heimbach, C. Hill, R. Ponte, and C. Wunsch, 2015: ECCO version 4: An integrated framework for non-linear inverse modeling and global ocean state estimation. Geosci. Model Dev., 8, 3071-3104, https://doi.org/ 10.5194/gmd-8-3071-2015.

Fukumori, I., O. Wang, I. Fenty, G. Forget, P. Heimbach, and R. Ponte, 2017: ECCO version 4 release 3. JPL Doc., ftp:// ecco.jpl.nasa.gov/Version4/Release3/doc/v4r3_summary.pdf.

—_, P. Heimbach, R. M. Ponte, and C. Wunsch, 2018: A dynamically-consistent ocean climatology and its temporal variations. Bull. Amer. Meteor. Soc., 99, 2107-2128, https:// doi.org/10.1175/BAMS-D-17-0213.1.

_- O. Wang, I. Fenty, G. Forget, P. Heimbach, and R. M. Ponte, 2019: ECCO Version 4 Release 4. https://ecco.jpl.nasa.gov/ drive/files/Version4/Release4/doc/v4r4_synopsis.pdf.

Gill, A. E., J. S. A. Green, and A. J. Simmons, 1974: Energy partition in the large-scale ocean circulation and the production of mid-ocean eddies. Deep-Sea Res., 21, 499-528, https:// doi.org/10.1016/0011-7471(74)90010-2.

Griffies, S. M., 2004: Fundamentals of Ocean Climate Models. Princeton University Press, 518 pp.

Heimbach, P., C. Wunsch, R. M. Ponte, G. Forget, C. Hill, and J. Utke, 2011: Timescales and regions of the sensitivity of Atlantic meridional volume and heat transport: Toward observing system design. Deep-Sea Res. II, 58, 1858-1879, https:// doi.org/10.1016/j.dsr2.2010.10.065.

, and Coauthors, 2019: Putting it all together: Adding value to the global ocean and climate observing systems with complete self-consistent ocean state and parameter estimates. Front. Mar. Sci., 6, 55, https://doi.org/10.3389/fmars.2019.00055.

$\mathrm{Hu}$, S., J. Sprintall, C. Guan, M. J. McPhaden, F. Wang, D. M. Hu, and W. Cai, 2020: Deep-reaching acceleration of global mean 
ocean circulation over the past two decades. Sci. Adv., 6, eaxx7727, https://doi.org/10.1126/sciadv.aax7727.

Huang, R. X., 2005: Available potential energy in the world's oceans. J. Mar. Res., 63, 141-158, https://doi.org/10.1357/0022240053693770.

Kalmikov, A., and P. Heimbach, 2014: A Hessian-based method for uncertainty quantification in global ocean state estimation. SIAM J. Sci. Comput., 36, S267-S295, https://doi.org/10.1137/130925311.

Kalnay, E., 2003: Atmospheric Modeling, Data Assimilation, and Predictability. Cambridge University Press, 341 pp.

Katija, K., 2012: Biogenic inputs to ocean mixing. J. Exp. Biol., 215, 1040-1049, https://doi.org/10.1242/jeb.059279.

Laepple, T., and P. Huybers, 2014: Global and regional variability in marine surface temperatures. Geophys. Res. Lett., 41, 25282534, https://doi.org/10.1002/2014GL059345.

Li, H., F. Xu, W. Zhou, D. Wang, J. Wright, Z. Liu, and Y. Lin, 2017: Development of a global gridded Argo data set with Barnes successive corrections. J. Geophys. Res. Oceans, 122, 866-889, https://doi.org/10.1002/2016JC012285.

Meyssignac, B., and Coauthors, 2019: Measuring global ocean heat content to estimate the Earth energy imbalance. Front. Mar. Sci., 6, 432, https://doi.org/10.3389/fmars.2019.00432.

Munk, W., 1981: Internal waves and small-scale processes. Evolution of Physical Oceanography: Scientific Surveys in Honor of Henry Stommel, B. A. Warren and C. Wunsch, Eds., MIT Press, 264-291, http://ocw.mit.edu/ans7870/resources/ Wunsch/wunschtext.htm.

and C. Wunsch, 1998: Abyssal recipes II: Energetics of tidal and wind mixing. Deep-Sea Res. I, 45, 1977-2010, https:// doi.org/10.1016/S0967-0637(98)00070-3.

Munk, W. H., and G. J. F. MacDonald, 1960: The Rotation of the Earth: A Geophysical Discussion. Cambridge University Press, 323 pp.

Olbers, D., J. Willebrand, and C. Eden, 2012: Ocean Dynamics. Springer, $704 \mathrm{pp}$.

Oort, A. H., L. A. Anderson, and P. Peixoto, 1994: Estimates of the energy cycle of the oceans. J. Geophys. Res., 99, 7665-7688, https://doi.org/10.1029/93JC03556.

Qiu, B., S. M. Chen, and N. Schneider, 2017: Dynamical links between the decadal variability of the Oyashio and Kuroshio extensions. J. Climate, 30, 9591-9605, https://doi.org/10.1175/ JCLI-D-17-0397.1.

Rascle, N., and F. Ardhuin, 2013: A global wave parameter database for geophysical applications. Part 2: Model validation with improved source term parameterization. Ocean Modell., 70, 174-188, https://doi.org/10.1016/j.ocemod.2012.12.001.

,,-- P. Queffeulou, and D. Croizé-Fillon, 2008: A global wave parameter database for geophysical applications. Part 1: Wave-current-turbulence interaction parameters for the open ocean based on traditional parameterizations. Ocean Modell., 25, 154-171, https://doi.org/10.1016/j.ocemod.2008.07.006.

Ray, R. D., and G. D. Egbert, 2018: Tides and satellite altimetry. Satellite Altimetry Over Oceans and Land Surfaces, D. Stammer and A. Cazenave, Eds., CRC Press, 427-458.
Reguero, B. G., I. J. Losada, and F. J. Méndez, 2019: A recent increase in global wave power as a consequence of oceanic warming. Nat. Commun., 10, 205, https://doi.org/10.1038/ s41467-018-08066-0.

Rimac, A., J.-S. Storch, and C. Eden, 2016: The total energy flux leaving the ocean's mixed layer. J. Phys. Oceanogr., 46, 18851900, https://doi.org/10.1175/JPO-D-15-0115.1.

Roemmich, D., and Coauthors, 2009: The Argo Program: Observing the global ocean with profiling floats. Oceanography, 22, 34-43, https://doi.org/10.5670/oceanog.2009.36.

Roquet, F., 2013: Dynamical potential energy: A new approach to ocean energetics. J. Phys. Oceanogr., 43, 457-476, https:// doi.org/10.1175/JPO-D-12-098.1.

Stammer, D., C. Wunsch, and K. Ueyoshi, 2006: Temporal changes in ocean eddy transports. J. Phys. Oceanogr., 36, 543-550, https://doi.org/10.1175/JPO2858.1.

Tailleux, R., 2013: Available potential energy and exergy in stratified fluids. Annu. Rev. Fluid Mech., 45, 35-58, https://doi.org/ 10.1146/annurev-fluid-011212-140620.

Thorpe, S., 2005: The Turbulent Ocean. Cambridge University Press, 439 pp.

von Storch, J. S., H. Sasaki, and J. Marotzke, 2007: Wind-generated power input to the deep ocean: An estimate using a $1 / 10^{\circ}$ general circulation model. J. Phys. Oceanogr., 37, 657-672, https://doi.org/10.1175/JPO3001.1.

Wang, J., G. R. Flierl, J. H. LaCasce, J. L. McClean, and A. Mahadevan, 2013: Reconstructing the ocean's interior from surface data. J. Phys. Oceanogr., 43, 1611-1626, https:// doi.org/10.1175/JPO-D-12-0204.1.

Wang, W., C. C. Qian, and R. X. Huang, 2006: Mechanical energy input to the world oceans due to atmospheric loading. Chin. Sci. Bull., 51, 327-330, https://doi.org/10.1007/s11434-006-0327-x.

Wilcox, R. R., 2010: Fundamentals of Modern Statistical Methods: Substantially Improving Power and Accuracy. 2nd ed. Springer, 258 pp.

Wunsch, C., 1997: The vertical partition of oceanic horizontal kinetic energy. J. Phys. Oceanogr., 27, 1770-1794, https://doi.org/10.1175/ 1520-0485(1997)027<1770:TVPOOH >2.0.CO;2.

— 2006: Discrete Inverse and State Estimation Problems: With Geophysical Fluid Applications. Cambridge University Press, $371 \mathrm{pp}$.

- 2016: Global ocean integrals and means, with trend implications. Annu. Rev. Mar. Sci., 8, 1-33, https://doi.org/10.1146/ annurev-marine-122414-034040.

_ 2018: Towards determining uncertainties in global oceanic mean values of heat, salt, and surface elevation. Tellus, $\mathbf{7 0 A}$, 1-14, https://doi.org/10.1080/16000870.2018.1471911.

_ 2020: On multi-year ocean thermal variability. Tellus, in press.

Young, I. R., and A. Ribal, 2019: Multiplatform evaluation of global trends in wind speed and wave height. Science, 364, 548-552, https://doi.org/10.1126/science.aav9527. 